\title{
CMEARTICLE
}

\section{Isolated right ventricle infarction}

Jia Wei $\underline{\text { Woo }}^{1}$, MBBS, MRCP, William Kong ${ }^{1}$, MBChB, FRACP, Anand $\underline{\text { Ambhore }}^{1}$, MD, MRCP, Saurabh $\underline{R a s t o g i}^{2}$, ABIM (Cardiology), ABIM (Interventional Cardiology), Kian Keong $\underline{\text { Poh }}^{1}$, MBBChir, FACC, Poay Huan Loh ${ }^{1}$, MD, FRCP

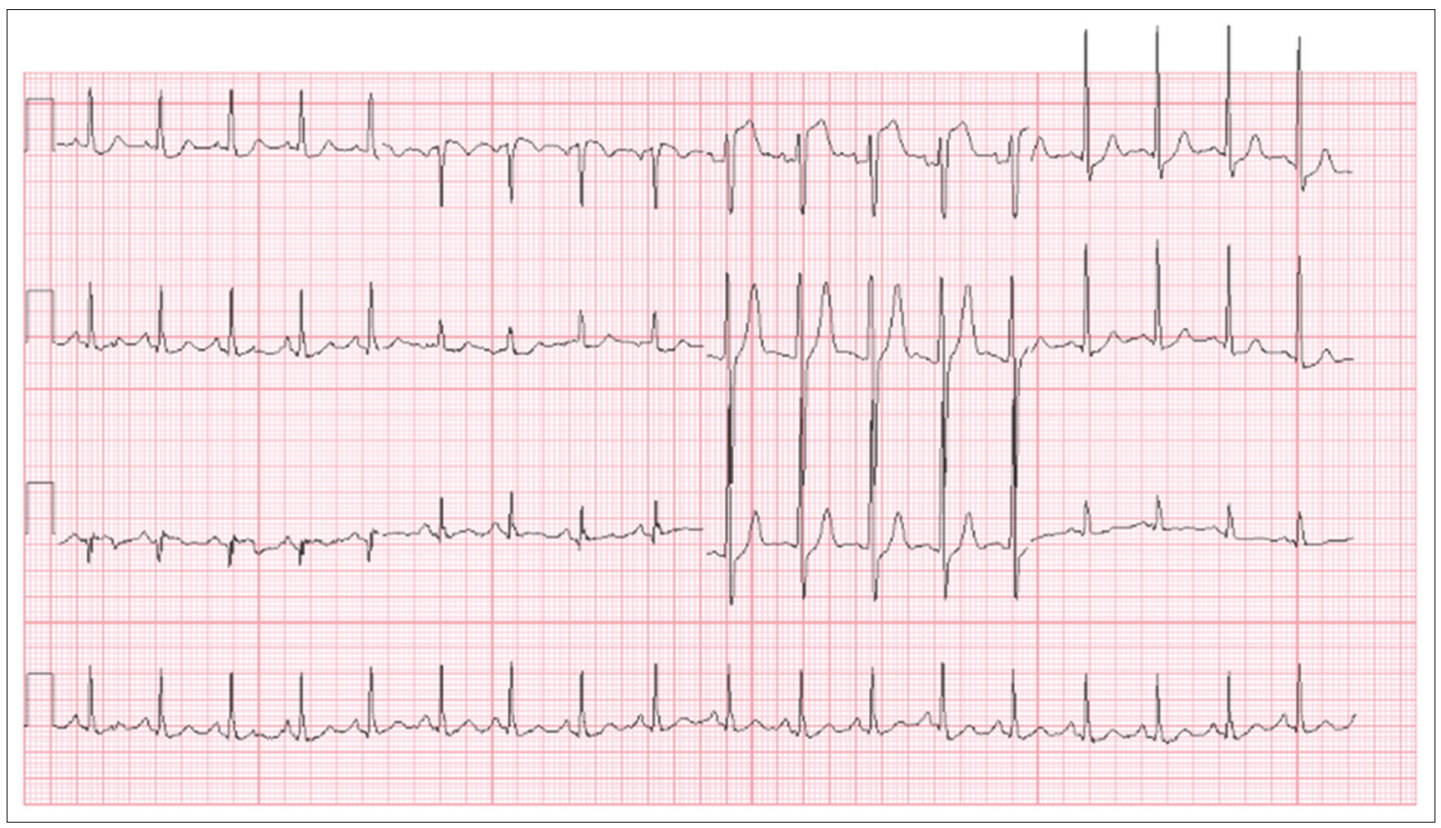

Fig. 1 Case 1: Patient's ECG on presentation.

\section{CASE 1}

\section{CLINICAL PRESENTATION}

A 42-year-old man with no significant past medical history or known cardiovascular risk factors had an out-of-hospital cardiac arrest while jogging. He received bystander cardiopulmonary resuscitation (CPR) immediately. Within five minutes of his collapse, paramedics arrived and used an automatic external defibrillator (AED) on him. Spontaneous circulation was re-established after the second cycle of CPR, with spontaneous breathing effort. He was taken to the local hospital emergency department and intubated, as he did not regain consciousness. What does the electrocardiogram (ECG) in Fig. 1 show?

\section{ECG INTERPRETATION}

The initial ECG showed sinus rhythm (Fig. 1). There was marked ST elevation in lead V1 and mild ST elevation in lead aVR. There was subtle ST depression from leads V3 to V5 and minimal ST elevation in lead III.

\section{CLINICAL COURSE}

Emergency coronary angiography revealed a proximal thrombotic occlusion in a large right ventricular (RV) branch of the right coronary artery (Fig. 2a). Balloon angioplasty was performed and a drug-eluting stent was implanted (Fig. 2b). Subsequently, the patient was managed with therapeutic hypothermia. Right-sided ECG leads obtained following coronary intervention showed subtle ST elevation (Fig. 3). Echocardiography showed preserved left ventricular systolic function. However, the tricuspid annular plane systolic excursion (TAPSE) in M-mode was $13 \mathrm{~mm}$, while RV systolic excursion velocity on tissue Doppler was $8 \mathrm{~cm} / \mathrm{second}$. These were consistent with moderate RV systolic dysfunction (Fig. 4).

On the day of admission, the patient was hypotensive with systolic blood pressure in the range of $70-80 \mathrm{mmHg}$. He required administration of multiple fluid boluses that amounted to $5 \mathrm{~L}$ in total in order to improve his preload and, hence, his blood pressure. Three days later, the patient was successfully weaned off therapeutic hypothermia and extubated. His hospital stay was uncomplicated and without any further arrhythmias. He made a complete neurological recovery and was discharged ten days after admission.

${ }^{1}$ Department of Cardiology, National University Heart Centre Singapore, National University Health System, ${ }^{2}$ Department of Cardiology, Ng Teng Fong General Hospital, Singapore Correspondence: Dr Jia Wei Woo, Registrar, Department of Cardiology, National University Heart Centre Singapore, National University Health System, 5 Lower Kent Ridge Road, Singapore 119074. Jia_wei_woo@nuhs.edu.sg 

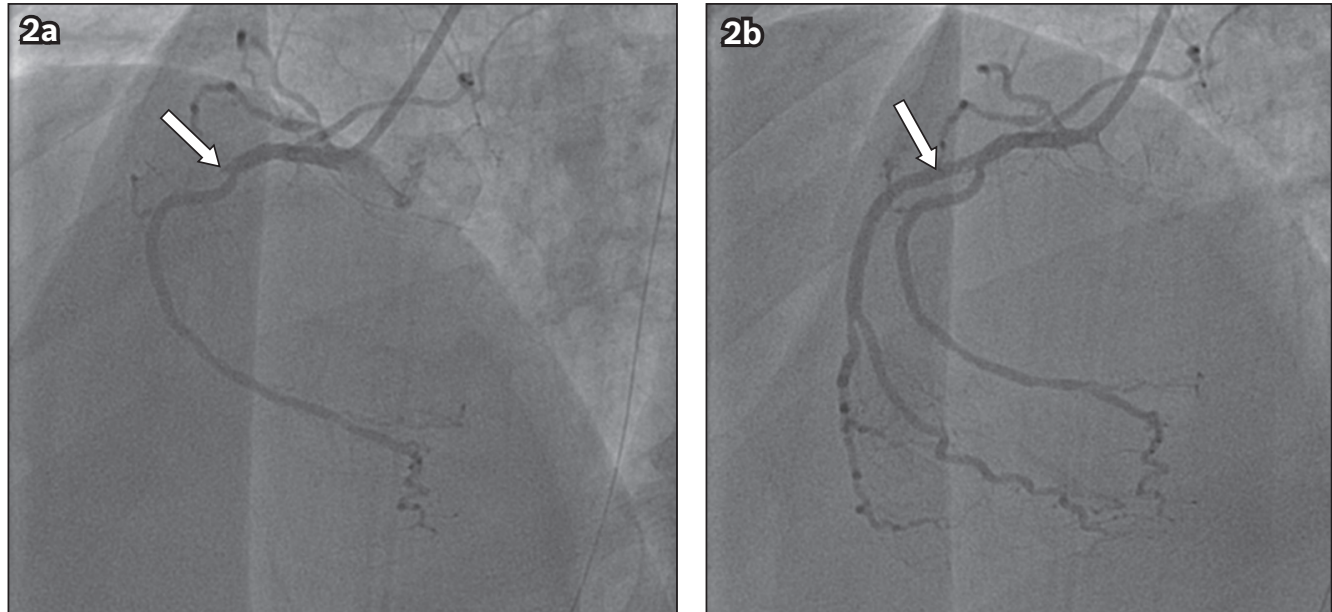

Fig. 2 Case 1: (a) Initial coronary angiogram shows the proximal occlusion of the right ventricular (RV) branch of the right coronary artery (arrow). (b) Coronary angiogram shows good flow into the RV branch of the right coronary artery post angioplasty (arrow).

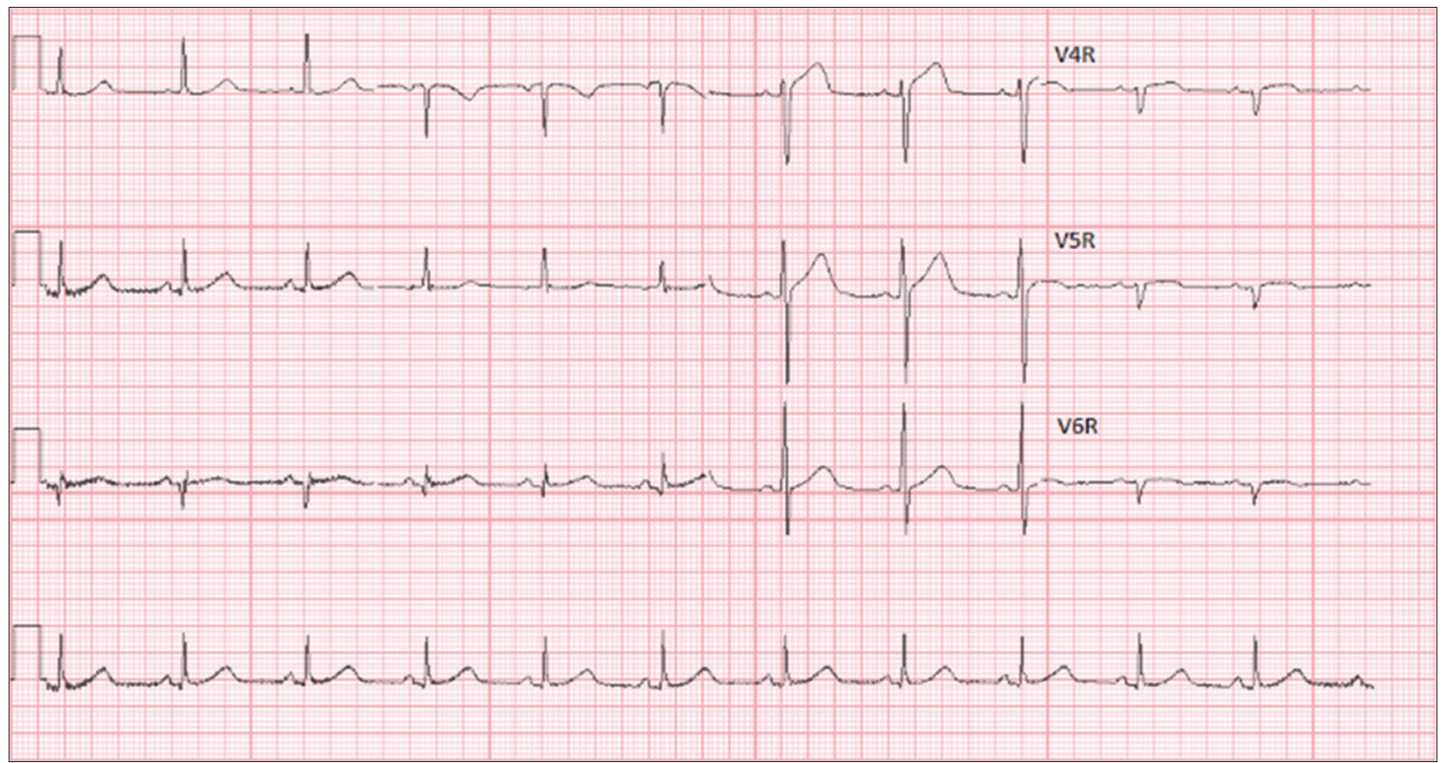

Fig. 3 Case 1: Right-sided chest leads of the patient show subtle ST elevation in V4R, V5R and V6R.
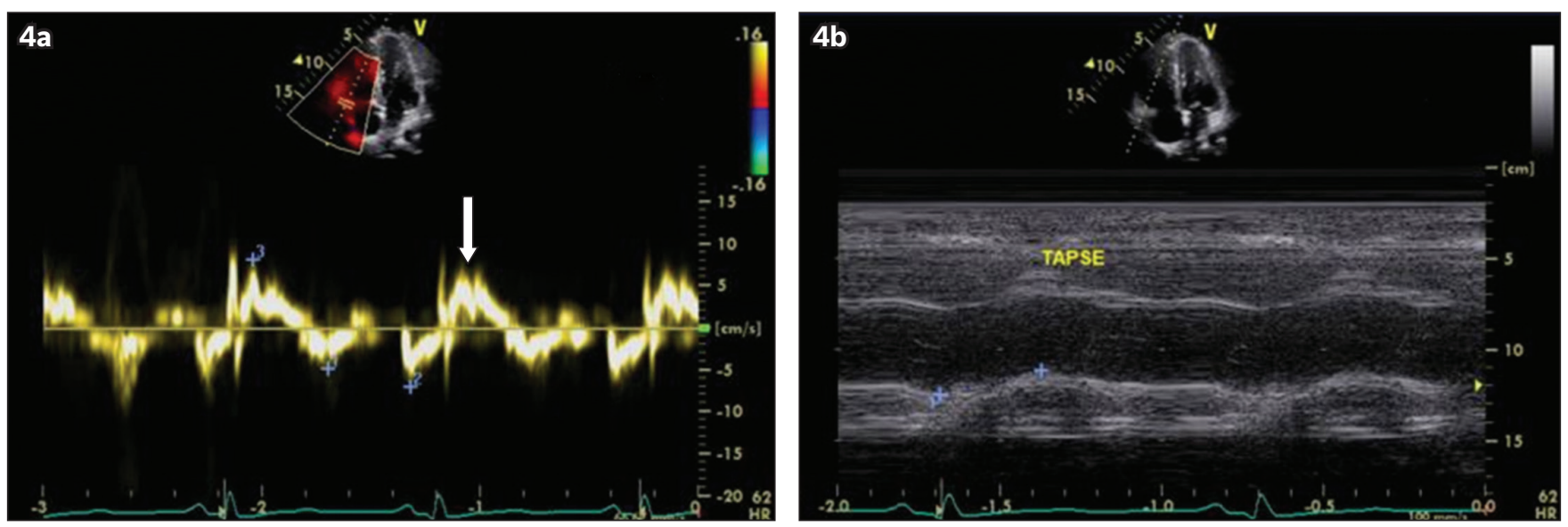

Fig. 4 Case 1: Echocardiograms show (a) right ventricular (RV) systolic excursion velocity of $8 \mathrm{~cm} /$ second (arrow), suggesting moderate RV systolic dysfunction; and (b) tricuspid annular plane systolic excursion of $13 \mathrm{~mm}$, indicating mild to moderate reduction in RV systolic function.

\section{CASE 2}

\section{CLINICAL PRESENTATION}

A 39-year-old man, who was a hepatitis B carrier without other past medical history, had an out-of-hospital cardiac arrest while working in a food centre. He was started on bystander CPR immediately until paramedics arrived. His initial rhythm was ventricular fibrillation. The resuscitation attempt continued while he was being transported to the hospital. Three shocks were 


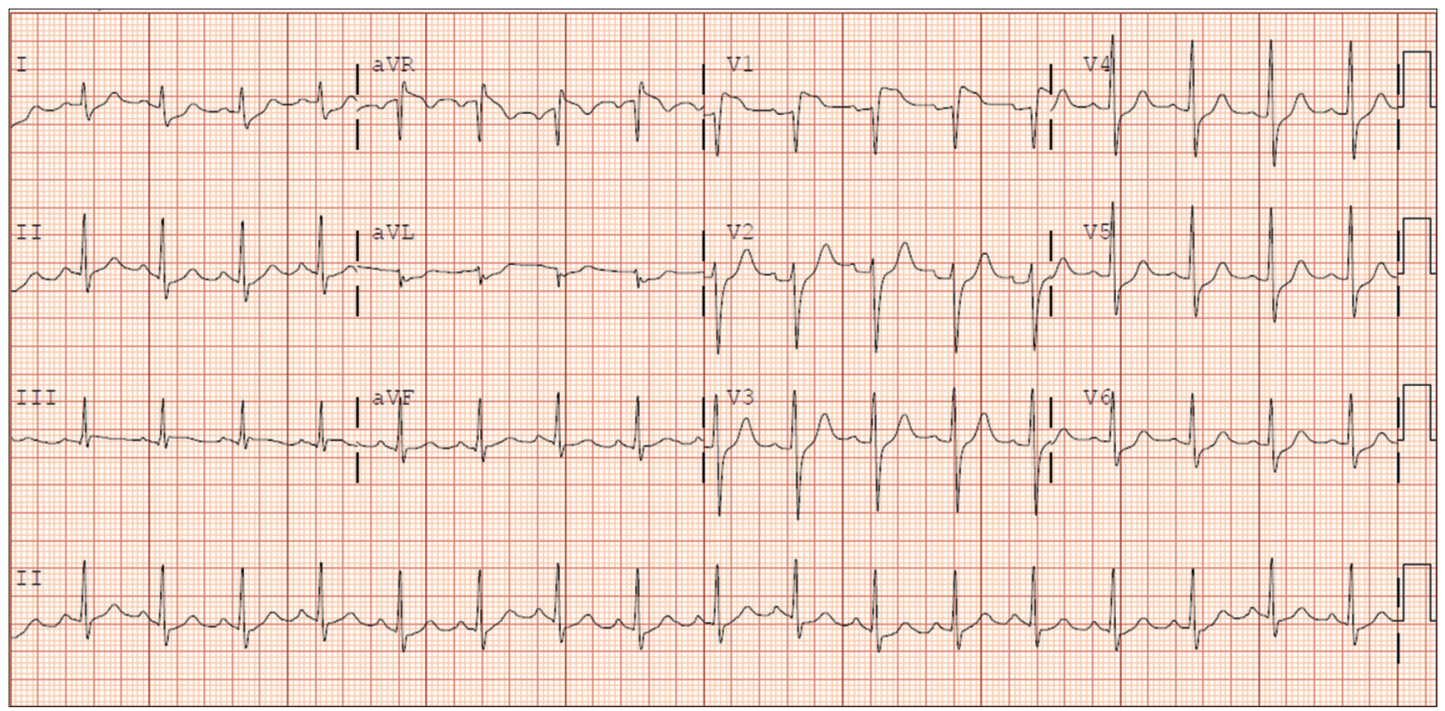

Fig. 5 Case 2: Initial ECG of the patient shows ST elevation in V1 that is higher than the ST elevation in aVR.
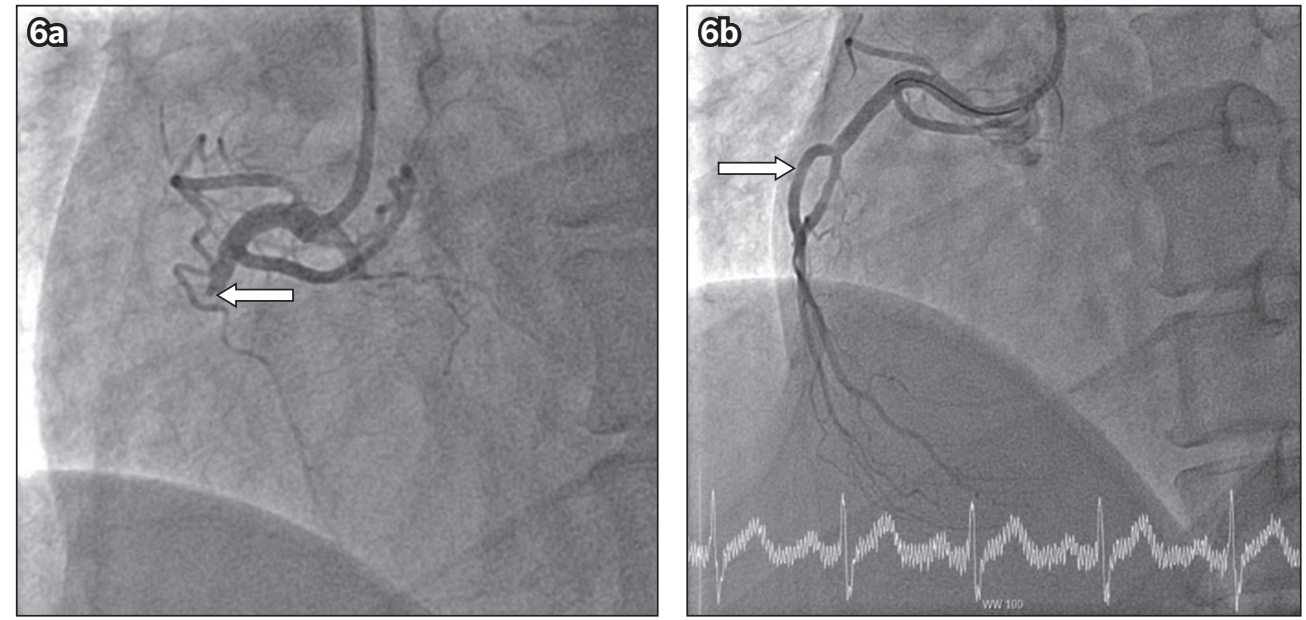

Fig. 6 Case 2: (a) Initial coronary angiogram shows occlusion of the proximal non-dominant right coronary artery (RCA; arrow). (b) Post-intervention coronary angiogram shows good flow in the proximal non-dominant RCA (arrow).

given before the patient had a return of spontaneous circulation on arrival at the hospital. He was alert and conversant when he was assessed in the emergency department.

\section{ECG INTERPRETATION}

The initial ECG (Fig. 5) showed ST elevation in leads V1 and aVR and diffuse ST depression from $\mathrm{V} 2$ to $\mathrm{V} 6$, as well as in leads I and aVL. ST elevation was more marked in V1 than aVR. No ST changes were seen in the inferior leads.

\section{CLINICAL COURSE}

Emergency coronary angiography showed an occlusion in the non-dominant right coronary artery just proximal to the origin of the RV branch (Fig. 6a). Intervention with balloon angioplasty and a drug-eluting stent produced good results (Fig. 6b). Echocardiography done the next day showed preserved left ventricular systolic function. However, the patient's TAPSE was $17 \mathrm{~mm}$ and RV systolic excursion velocity on tissue Doppler was $12 \mathrm{~cm} / \mathrm{second}$, which were consistent with normal RV function but at the lower limit of normal. His hospital course was uncomplicated and he was discharged three days after admission.

\section{DISCUSSION}

In both patients, the presenting ECG, coronary angiogram and echocardiography findings were consistent with an isolated RV infarction. As isolated RV infarction is rare, it is not uncommon to misinterpret it to be an infarction of the anterior left ventricular wall, or even a left main stem occlusion, because of ST changes in the anterior leads V1-V3.$^{(1-3)}$ However, in these cases, ST elevation in V1 was more marked than ST elevation in aVR, which differentiates acute RV infarction from anterior wall infarction or left main coronary artery infarction. ${ }^{(1,3)}$ This might be because anatomically, lead V1 is located directly over the right ventricle, thus, V1 ST elevation is also greater than ST elevation in V2 and V3 in both cases.

Obtaining right-sided ECG V4R, V5R and V6R leads may aid in the diagnosis, since ST elevation and $\mathrm{Q}$ waves in $\mathrm{V} 4 \mathrm{R}$ may be diagnostic for isolated RV infarction. ${ }^{(3,4)}$ On the other hand, although a posterior ECG (leads V7-V9) has utility to detect 
infarction in the posterior circulation of the left ventricle, it would not be useful in diagnosing an RV infarction. It should also be noted that the anterior chest leads are perpendicular to the limb leads and should not cause reciprocal inferior ST depression in the limb leads, unless there is widespread left ventricular ischaemia causing ST depression. The inferior limb leads in II, III and aVF are, in fact, reciprocal to limb leads I and aVL. ${ }^{(4)}$

The patient's coronary angiogram in Case 1 showed occlusion in the RV branch, which was consistent with the ECG finding of acute isolated RV infarction. More commonly, classic inferior ST elevation myocardial infarction presenting with RV failure would have an occlusion in the proximal right coronary artery proximal to the RV branch. However, in Case 1, the main right coronary artery branch was spared, while the occlusion was confined to the RV branch, causing an isolated RV infarction. The patient in Case 2 had a proximal occlusion of a non-dominant right coronary artery. As the non-dominant right coronary artery predominantly supplies the right ventricle, his presentation mimicked that of an isolated RV infarction.

The involvement of the myocardium during occlusion of the right coronary artery is typically greater in a right-dominant coronary system when the posterior descending artery and the posterolateral artery originate from the right coronary artery. In a minority of patients with a left-dominant circulation, where the posterior descending artery and the posterolateral artery branch off from the left circumflex artery, the right coronary artery is non-dominant. A non-dominant right coronary artery may have limited contribution to the left ventricle, as observed with the patient in Case 2.

An important complication to recognise with RV infarction is RV failure. As such, adequate preload is vital to maintain satisfactory cardiac output. The patient in Case 1 had myocardial infarction while he was jogging and was thus likely to have lost a substantial amount of fluid through perspiration. He was dehydrated and intravascularly depleted at presentation. This fluid deficit had to be taken into account during the initial resuscitation in order to maintain his preload and cardiac output. In patients who are hypotensive with RV failure, the first-line treatment is augmentation of RV preload with boluses of intravenous fluids. ${ }^{(5)}$ Thus, nitrates are not haemodynamically favourable in these patients, as they would cause venodilatation, which may reduce the RV preload. ${ }^{(3)}$ As these patients are usually responsive to an increase in preload from intravenous fluids, inotropes and mechanical support with devices such as an intra-aortic balloon pump are not considered as first-line therapy. ${ }^{(5)}$ Maintaining adequate RV preload was less important for the patient in Case 2, as he had not been engaged in intense physical activity that led to substantial fluid loss. Left ventricular systolic function is usually not an issue in isolated RV infarction, as seen in both patients.

An isolated RV infarction may be proarrhythmic, and patients may present with out-of-hospital cardiac arrest, as seen in both our patients. It was previously postulated that RV infarction causes sympathetic and vagal denervation at the right ventricular outflow tract (RVOT) and lateral and septal sides of the viable peri-infarct area. Autonomic denervation in the RVOT may contribute to the development of ventricular tachyarrhythmias during the acute phase of RV infarction. ${ }^{(6)}$

Although isolated RV infarction has been described to have a good prognosis, ${ }^{(1-3,7)}$ the prognosis following cardiac arrest in these cases was also dependent on early CPR. Both patients had a short no-flow time, which is defined as time without chest compression during the first period of cardiac arrest, as they received almost immediate bystander CPR. While the patient in Case 2 was alert and talking on arrival at the emergency department, the patient in Case 1 remained unconscious on arrival at the hospital. Thus, the patient in Case 1 was also given therapeutic hypothermia, which could have contributed to a favourable neurological recovery. ${ }^{(8,9)}$ Although the rate of bystander CPR in Singapore remained at a low of about $20 \%$ until 2011, the implementation of dispatcher-assisted CPR by the Singapore Civil Defence Force has led to a rise in bystander CPR rates, to $49 \%$ in $2015 . .^{(10-12)}$ Thus, dispatcher-assisted CPR may improve outcomes in patients with out-of-hospital cardiac arrest in Singapore. ${ }^{(13)}$

One possible reason that laypersons may be averse to performing bystander CPR is that mouth-to-mouth ventilation is still included in the standard Basic Cardiac Life Support (BCLS) protocol, although evidence suggests that it does little to improve outcomes. ${ }^{(14,15)}$ Making mouth-to-mouth ventilation optional in the standard BCLS protocol taught to members of the public may encourage more bystander CPRs. Furthermore, using a simpler protocol may boost confidence. This would translate to improved outcomes for patients suffering from out-of-hospital cardiac arrest in Singapore. ${ }^{(11-14,16)}$

While $82.6 \%$ and $57.2 \%$ of Singaporeans feel that all adults should be trained in CPR and AED, respectively, the actual proportion of Singaporeans who are trained in these is only $34.3 \%$ and $31.4 \%$, respectively. ${ }^{(17)}$ In order to enhance training in CPR and AED, the National Resuscitation Council has set up a system for monitoring and maintaining standards of life support training, leading to a large increase in the number of training centres for BCLS. ${ }^{(18)}$ Bystander CPR has also been promoted through community-based training programmes. These initiatives would likely lead to improved outcomes in future for individuals with out-of-hospital cardiac arrests. ${ }^{(18)}$

ABSTRACT We described two patients who were successfully resuscitated from out-of-hospital cardiac arrest. Their ECGs showed ST elevations in V1 and aVR, as well as diffuse ST depression. Their ST elevation in V1 was noted to be greater than in aVR. While one patient was found to have an occlusion of the right ventricular (RV) branch of the right coronary artery, the other was found to have an occlusion of a proximal nondominant right coronary artery supplying the RV branch. Successful primary percutaneous coronary intervention was performed for each patient with angioplasty and implantation of a drug-eluting stent. Both patients made good physical and neurological recovery.

Keywords: ECG, isolated right ventricular infarction, out-of-hospital cardiac arrest, $R V$ branch occlusion 


\section{REFERENCES}

1. Finn AV, Antman EM. Images in clinical medicine. Isolated right ventricular infarction. N Engl J Med 2003; 349:1636.

2. Turkoglu S, Erden M, Ozdemir M. Isolated right ventricular infarction due to occlusion of the right ventricular branch in the absence of percutaneous coronary intervention. Can J Cardiol 2008; 24:793-4.

3. Chalhal AA, Kim Y, Borg AN, Al-Najjar Y. Primary angioplasty for infarction due to isolated right ventricular artery occlusion. World J Cardiol 2014; 6:1223-6.

4. Poh KK, Tan HC, Teo SG. ECG ST segment elevation in patients with chest pain. Singapore Med J 2011; 52:3-7.

5. Tan AT, Chia BL, Ee BK, Choo M. Right ventricular infarction - clinical haemodynamic, echocardiographic and therapeutic considerations. Singapore Med J 1982; 23:102-6.

6. Elvan A, Zipes DP. Right ventricular infarction causes heterogeneous autonomic denervation of the viable peri-infarct area. Circulation 1998; 97:484-92.

7. Assali AR, Teplitsky I, Ben-Dor I, et al. Prognostic importance of right ventricular infarction in an acute myocardial infarction cohort referred for contemporary percutaneous reperfusion therapy. Am Heart J 2007; 153:231-7.

8. Holzer M, Bernard SA, Hachimi-Idrissi S, et al; Collaborative Group on Induced Hypothermia for Neuroprotection After Cardiac Arrest. Hypothermia for neuroprotection after cardiac arrest: systematic review and individual patient data meta-analysis. Crit Care Med 2005; 33:414-8.

9. Leong $\mathrm{SH}, \mathrm{Chan} \mathrm{E}, \mathrm{Ho} \mathrm{BC}$, et al. Therapeutic temperature management (TTM): post-resuscitation care for adult cardiac arrest, with recommendations from the
National TTM Workgroup. Singapore Med J 2017; 58:408-10.

10. Charles RA, Lateef F, Anantharaman V. Strengthening links in the "chain of survival"; a Singapore perspective. Hong Kong J Emerg Me 2002; 9:121-5.

11. Ong ME, Shin SD, De Souza NN, et al. Outcomes for out-of-hospital cardiac arrests across 7 countries in Asia: The Pan Asian Resuscitation Outcomes Study (PAROS). Resuscitation 2015; 96:100-8.

12. Ng YY, Leong $\mathrm{SH}$, Ong ME. The role of dispatch in resuscitation. Singapore Med J 2017; 58:449-52.

13. Harjanto S, Na MX, Hao Y, et al. A before-after interventional trial of dispatcherassisted cardio-pulmonary resuscitation for out-of-hospital cardiac arrests in Singapore. Resuscitation 2016; 102:85-93.

14. Fong YT, Anantharaman V, Lim SH, Leong KF, Pokkan G. Mass cardiopulmonary resuscitation 99--survey results of a multi-organisational effort in public education in cardiopulmonary resuscitation. Resuscitation 2001; 49:201-5.

15. Hallstrom A, Cobb L, Johnson E, Copass M. Cardiopulmonary resuscitation by chest compression alone or with mouth-to-mouth ventilation. $\mathrm{N}$ Engl J Med 2000; 342:1546-53.

16. Song KJ, Shin SD, Park CB, et al. Dispatcher-assisted bystander cardiopulmonary resuscitation in a metropolitan city: a before-after population based study. Resuscitation 2014; 85:34-41.

17. Ong ME, Quah JL, Ho AF, et al. National population based survey on the prevalence of first aid, cardiopulmonary resuscitation and automated external defibrillator skills in Singapore. Resuscitation 2013; 84:1633-6.

18. Anantharaman V. The National Resuscitation Council, Singapore, and 34 years of resuscitation training: 1983 to 2017. Singapore Med J 2017; 58:418-23. 


\section{SINGAPORE MEDICAL COUNCIL CATEGORY 3B CME PROGRAMME} (Code SMJ 201903B)

Question 1. This finding supports the diagnosis of right ventricular (RV) infarction:

(a) ST elevation in the right-sided electrocardiogram (ECG) leads V4R-V6R.

(b) ST elevation in V1 is greater than ST elevation in aVR.

(c) ST elevation in the posterior ECG leads.

(d) ST elevation in V1 is greater than that in V2 and V3.

Question 2. The following is appropriate first-line management for a patient who is hypotensive with RV failure:

(a) Inotropes to support blood pressure.

(b) Fluid boluses to maintain right ventricular preload.

(c) Nitrates to relieve angina.

(d) Insertion of intra-aortic balloon pump.

Question 3. Regarding an out-of-hospital cardiac arrest:

(a) Downtime is defined as time without chest compression during the first period of cardiac arrest.

(b) No-flow time is defined as time from collapse to return of spontaneous circulation.

(c) The implementation of dispatcher-assisted cardiopulmonary resuscitation (CPR) by the Singapore Civil Defence Force has led to a rise in bystander CPR rates.

(d) Mouth-to-mouth ventilation has led to improved outcomes for patients with out-of-hospital cardiac arrests.

Question 4. In right coronary artery myocardial infarction:

(a) Left ventricular systolic failure is one of the common complications.

(b) RV failure is one of the common complications.

(c) RV function for patients with myocardial infarction can be estimated from tricuspid annular plane systolic excursion.

(d) Tissue Doppler is not useful in estimating RV function.

Question 5. Which of the following statements is true about coronary anatomy?

(a) In an isolated RV infarction, the artery involved is the RV branch, which branches off from the proximal part of the right coronary artery.

(b) A non-dominant right coronary artery would typically support more myocardium compared to a dominant right coronary artery.

(c) In a left-dominant coronary artery circulation, the posterior descending artery branches off from the right coronary artery.

(d) In a left-dominant coronary artery circulation, the posterior descending artery branches off from the left circumflex artery.

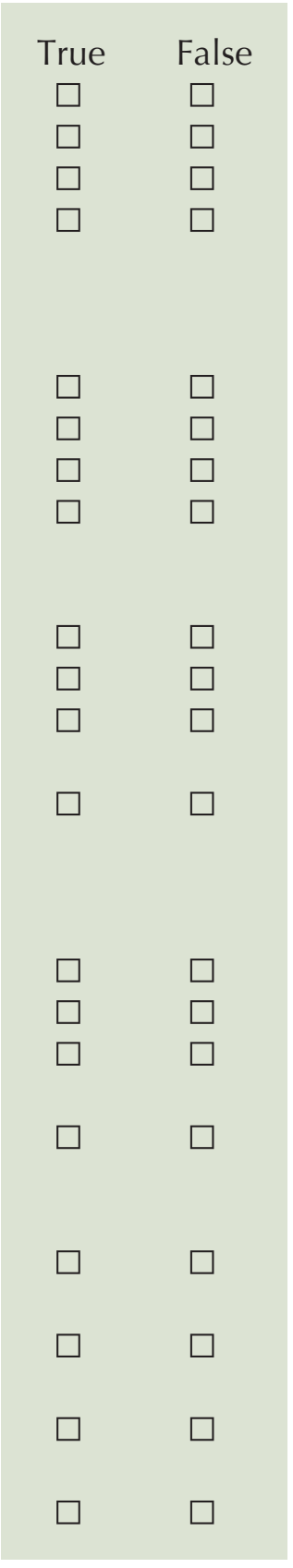

\section{Doctor's particulars:}

Name in full:

MCR no.:

Specialty:

Email:

\footnotetext{
SUBMISSION INSTRUCTIONS:

Visit the SMJ website: http://www.smj.org.sg/current-issue and select the appropriate quiz. You will be redirected to the SMA login page.

For SMA member: (1) Log in with your username and password (if you do not know your password, please click on 'Forgot your password?'). (2) Select your answers for each quiz and click 'Submit'.

For non-SMA member: (1) Create an SMJ CME account, or login with your SMJ CME username and password (for returning users). (2) Make payment of SGD 21.40 (inclusive of $7 \%$ GST) via PayPal to access this month's quizzes. (3) Select your answers for each quiz and click 'Submit'.

RESULTS:

(1) Answers will be published online in the SMJ May 2019 issue. (2) The MCR numbers of successful candidates will be posted online at the SMJ website by 9 May 2019. (3) Passing mark is $60 \%$. No mark will be deducted for incorrect answers. (4) The SMJ editorial office will submit the list of successful candidates to the Singapore Medical Council. (5) One CME point is awarded for successful candidates. (6) SMC credits CME points according to the month of publication of the CME article (i.e. points awarded for a quiz published in the December 2018 issue will be credited for the month of December 2018, even if the deadline is in January 2019).

Deadline for submission (March 2019 SMJ 3B CME programme): 12 noon, 2 May 2019.
} 